\title{
NORMAL CLOSURES OF POWERS OF DEHN TWISTS IN MAPPING CLASS GROUPS
}

\author{
by STEPHEN P. HUMPHRIES
}

(Received 3 April, 1991; revised 5 September, 1991)

1. Let $F=F(g, n)$ be an oriented surface of genus $g \geq 1$ with $n<2$ boundary components and let $M(F)$ be its mapping class group. Then $\mathrm{M}(\mathrm{F})$ is generated by Dehn twists about a finite number of non-bounding simple closed curves in $F([6,5])$. See $[1]$ for the definition of a Dehn twist. Let $e$ be a non-bounding simple closed curve in $F$ and let $E$ denote the isotopy class of the Dehn twist about $e$. Let $N$ be the normal closure of $E^{2}$ in $M(F)$. In this paper we answer a question of Birman [1, Qu 28 page 219]:

THEOREM 1. The subgroup $N$ is of finite index in $M(F)$.

In fact we prove somewhat more:

THEOREM 2. If $F$ is closed and has genus two or three, then the normal closure of $E^{3}$ is of finite index in $M(F)$.

THEOREM 3. If $F$ has genus two and has a single boundary component, then the normal closure of $E^{2}$ or $E^{3}$ is of finite index in $M(F)$.

On the other hand we prove:

THEOREM 4. If $F$ has genus two and has $n \geq 0$ boundary components, then the normal closure of $E^{k}$ is of infinite idex in $M(F)$ for all $k>3$.

The case $g=1, n=0$ gives the group $M=S L(2, \mathbb{Z})[1]$ and a Dehn twist is represented by a matrix conjugate to the parabolic matrix $E=\left(\begin{array}{ll}1 & 1 \\ O & 1\end{array}\right)$. Let $N^{k}$ be the normal closure of $E^{k}$. Then $N^{k}$ is of index $6,24,48,120$ for $k=2,3,4,5$ (respectively) and is of infinite index if $n>5$ [9]. The case $g=1, n=1$ gives the group $M=B_{3}$, the braid group on 3 strings [1] and a Dehn twist is represented by one of the standard braid generators $\sigma$. Let $N^{k}$ be the normal closure of $\sigma^{k}$. Then $N^{k}$ is of index 6, 24, 96, 600 for $k=2,3,4,5$ (respectively) and is of infinite index if $n>5$.

2. Proof of Theorem 1. Let $F=F(g, 0), g>1$. Let $S p(2 g, R)$ be the symplectic group of rank $2 g$ matrices with coefficients in the ring $R=\mathbb{Z}$ or $\mathbb{Z} / m \mathbb{Z}$. If we think of the underlying symplectic space on which this symplectic group acts as being the homology group $H_{1}(F, R)$ with its natural symplectic form coming from the algebraic intersection number, then we have a natural map $M(F) \rightarrow S p(2 g, R)$ which is actually onto [7 p. 178]. By a $k$-chain of simple closed curves in $F$ we will mean a sequence $c_{0}, c_{1}, c_{2}, \ldots, c_{k-1}$ of homologically independent simple closed curves in $F$ such that $c_{i}$ and $c_{j}$ intersect if and only if $|i-j|=1$ and then only once geometrically. Theorem 1 will follow from:

Glasgow Math. J. 34 (1992) 313-317. 
Proposition 2.1. Let $F=F(g, 0)$ and let $N$ be the normal closure of $E^{2}$ in $M(F)$. Then $N$ is the kernel of the natural map

$$
\varphi: M(F) \rightarrow S p(2 g, \mathbb{Z} / 2 \mathbb{Z})
$$

Proof. An easy calculation shows that $N$ is contained in the kernel of the map $\varphi$. Let $\varphi^{\prime}: M(F) \rightarrow S p(2 g, \mathbb{Z})$ be the map giving the action of $M(F)$ on $H_{1}(F, \mathbb{Z})$. Then $\varphi^{\prime}$ is surjective ([7] p. 178) and $\varphi$ is the composite of epimorphisms

$$
M(F) \rightarrow \operatorname{Sp}(2 g, \mathbb{Z}) \rightarrow \operatorname{Sp}(2 g, \mathbb{Z} / 2 \mathbb{Z})
$$

We will prove (a) that $N$ contains the kernel of the first map and (b) that the image of $N$ in $S p(2 g, \mathbb{Z})$ is exactly the kernel of the second map. For (a) we note that by [10] the kernel $I_{g}$ of the map $\varphi^{\prime}$ is generated by (i) Dehn twists about bounding curves, and (ii) bounding pairs. Here a bounding pair is a product $G H^{-1}$, where $G$ and $H$ are Dehn twists about disjoint non-bounding simple closed curves 1 al $F$ which together bound in $F$. This kernel is called the Torelli group. To prove (a) it will suffice to show that $N$ contains all generators of types (i) and (ii). For generators of type (i) we will prove the following more general result:

LEMMA 2.2. Let $F$ be a surface of genus $g>0$ with at most one boundary component. Then the normal closure $N$ of $E^{2}$ contains the Dehn twists about all bounding curves.

Proof. First note ([6] or [1]) that if $C$ is any Dehn twist about a non-bounding curve in $F$, then there is an element $\alpha$ of $M(F)$ such that $\alpha E \alpha^{-1}=C$. It follows that $C^{2}$ belongs to $N$. Now note that if $d$ is a bounding curve in $F$ with Dehn twist $D$, then there is another bounding curve $d^{\prime}$ with Dehn twist $D^{\prime}$ such that $d^{\prime}$ bounds a surface of strictly smaller genus (possibly zero) than does $d$ and such that $d$ and $d^{\prime}$ together bound a genus 1 surface containing a 3-chain of simple closed curves $x, y z$ with Dehn twists $X, Y, Z$. Now $x$ and $z$ are disjoint curves and so $X$ and $Z$ commute. This fact and [6, Lemma 3] implies that

$$
D D^{\prime}=X Z Y X Z Y^{2} X Z Y X Z
$$

lies in $N$. It easily follows by induction that each such $D$ belongs to $N$. This proves the Lemma and shows that the subgroup generated by Dehn twists about bounding curves lies in $N$.

For generators of type (ii) we first note that for any bounding pair $B D^{-1}$ there is a $(2 g+1)$-chain $c_{0}, c_{1}, c_{2}, \ldots, c_{2 g}$ such that $b$ and $d$ only intersect some $c_{k}$ for fixed odd $k$ and then only once. Let

$$
w=C_{0} C_{1} C_{2} C_{3} \ldots C_{g-2} C_{g-1} C_{g}^{2} C_{g-1} C_{g-2} \ldots C_{1} C_{0}
$$

Then $w$ is an involution of $F[1]$ and satisfies $w(b)=d$. One first notes that $w$ belongs to $N$ and so $B w B^{-1} w^{-1}=B D^{-1}$ also belongs to $N$. This proves case (ii).

We next show that the image of $N$ in $S p(2 g, \mathbb{Z})$ is equal to the kernel of the natural $\operatorname{map} S p(2 g, \mathbb{Z}) \rightarrow S p(2 g, \mathbb{Z} / 2 \mathbb{Z})$. We note that the image of $D$ in $S p(2 g, \mathbb{Z})$ is a primitive symplectic transvection $T$ and that the normal closure of $T^{2}$ is a finite index in $\operatorname{Sp}(2 g, \mathbb{Z})$ since by [8] it is equal to the kernel of the map $\operatorname{Sp}(2 g, \mathbb{Z}) \rightarrow \operatorname{Sp}(2 g, \mathbb{Z} / 2 \mathbb{Z})$. Theorem 1 now follows. 
3. Proof of Theorem 2. Now suppose that $F=F(2,0)$ and let $N$ be the normal closure in $M(F)$ of $E^{3}$. We want to show that $N$ contains $I_{2}$. Again [10] shows that $I_{2}$ is generated by Dehn twists about bounding curves only, since there are no bounding pairs in this case.

LemMA 3.1. Let $F$ be a surface of genus $g$ with or without boundary and let $N$ be the normal closure in $M(F)$ of $E^{3}$. If $D$ is the Dehn twist about a bounding curve $d$ in $F$ which bounds a genus 1 subsurface, then $D$ lies in $N$.

Proof. By the hypothesis we see that there is a 2-chain $a, b$ in $F$ such that $d$ is isotopic to the boundary of a tubular neighbourhood of $a \cup b$. Then one calculates that $D=(A B A)^{4}$. Now $A B A=B A B$ and so

$$
\begin{aligned}
D & =A B A A B A A B A A B A=A B A A B A B A B A B A \\
& =A B A A A B A A B A B A=(A B) A A A(A B)^{-1} A B B A A B A B A \\
& =(A B) A A A(A B)^{-1} A B B A B A B B A=(A B) A A A(A B)^{-1} A B B B A B B B A
\end{aligned}
$$

which clearly belongs to $N$.

Returning to the case where $g=2$ and $F$ is closed this lemma shows that $N$ contains all Dehn twists about bounding curves and so contains $I_{2}$. Again [8] shows that the image of $N$ in $S p(4, \mathbb{Z} / 3 \mathbb{Z})$ is equal to the kernel of the natural map $S p(4, \mathbb{Z}) \rightarrow S p(4, \mathbb{Z} / 3 \mathbb{Z})$.

Now suppose that $F=F(3,0)$ and that $N$ is the normal closure of $E^{3}$. To show that $N$ has finite index in $M(F)$ it will suffice to show (i) that $I_{3} \cap N$ has finite index in $I_{3}$ and (ii) that the image of $N$ in $M(F) / I_{3}=S p(6, \mathbb{Z})$ has finite index. In fact this latter fact again follows from [8]. For (i) we note that by [3] there is a map $\tau: I_{3} \rightarrow A$ where $A$ is a free abelian group of rank 14 and by [4] the kernel $K$ of $\tau$ is the subgroup generated by twists on bounding curves. Since $F$ has genus three and is closed we see that any bounding curve bounds a surface of genus 1 and so Lemma 3.1 shows that any Dehn twist about a bounding curve lies in $N$. Thus $N$ contains $K$ and we now need only show (i)' $\tau\left(I_{3} \cap N\right)$ has finite index in $A$. Since $I_{3} / K$ is generated by the images of bounding pairs (i)' will follow from the fact that if $B D^{-1}$ is a bounding pair, then $\left(B D^{-1}\right)^{3}=B^{3} D^{-3}$ belongs to $N$. This shows that in fact

$$
I_{3} /\left(I_{3} \cap N\right)=\tau\left(I_{3}\right) / \tau\left(I_{3} \cap N\right)=(\mathbb{Z} / 3 \mathbb{Z})^{14}
$$

and so $M(F) / N$ is an extension of $S p(6, \mathbb{Z} / 3 \mathbb{Z})$ by $(\mathbb{Z} / 3 \mathbb{Z})^{14}$. This concludes the proof of Theorem 2.

4. Proof of Theorem 3. Let $F=F(2,1)$ be a genus 2 surface with a single boundary component and let $N$ be the normal closure of $E^{2}$. Let $T$ be the subgroup of the Torelli subgroup $I_{2,1}$ generated by the Dehn twists about bounding curves. Let $T_{i}, i=1,2$, be the subgroup of $T$ generated by the Dehn twists about bounding curves of genus $i$. Here the genus of a bounding curve is the genus of the surface that it bounds. Clearly $T$ is generated by $T_{1}$ and $T_{2}$, since $F$ has genus 2 . Note that there is only one (isotopy class of) bounding closed curve of genus 2 , namely the curve parallel to the boundary component. It follows that $T_{2}$ is in the centre of $T$. Now by Lemma 2.2 we see that $N$ contains all of $T$. Again [4] shows that $T$ is the kernel of the map $\tau: I_{2,1} \rightarrow A$ where here $A$ is a free abelian 
group of rank 4. An argument similar to that in $\$ 3$ shows that

$$
I_{1,2} /\left(I_{2,1} \cap N\right)=\tau\left(I_{2,1}\right) / \tau\left(I_{2,1} \cap N\right)=(\mathbb{Z} / 2 \mathbb{Z})^{4} .
$$

This now shows that $M(F) / N$ is an extension of $S p(4, \mathbb{Z} / 2 \mathbb{Z})$ by $(\mathbb{Z} / 2 \mathbb{Z})^{4}$ and so is finite.

Let $F$ be a genus 2 surface with a single boundary component and let $N$ be the normal closure of $E^{3}$. By Lemma 3.1 we see that $N$ contains $T_{1}$ and so to conclude the argument we must show that some power of the generator of $T_{2}$ belongs to $N$. If $D^{\prime}$ is this generator, then by $\left(^{*}\right)$ we have

$$
D^{\prime}=D^{-1} X Z Y X Z Y^{2} X Z Y X Z,
$$

where $D$ is the Dehn twist about a genus 1 bounding curve not meeting $x, y$ or $z$. Thus

$$
D^{\prime k}=D^{-k}\left(X Z Y X Z Y^{2} X Z Y X Z\right)^{k} \text {, }
$$

for all $k$. Now $X, Y$ and $Z$ satisfy the "braid relations": $X Z=Z X, X Y X=Y X Y$, $Y Z Y=Z Y Z$ and if we now add in the relations $X^{3}=Y^{3}=Z^{3}=$ identity coming from $N$, then (by the Todd-Coxeter algorithm) we obtain a group of order $646=2^{3} 3^{4}$ in which the element $Z Y X Z Y^{2} X Z Y X Z$ has order 3. By Lemma 3.1 we see that $D^{3}$ is in $N$ and so $D^{\prime 3}$ belongs to $N$ as required. Thus $T /(T \cap N)$ is finite; in fact it is $\mathbb{Z} / 3 \mathbb{Z}$. It easily follows that $\tau\left(I_{2,1}\right) / \tau\left(I_{2,1} \cap N\right)$ is a finite abelian 3-group and so $M(F) / N$ is a finite extension of $S p(4, \mathbb{Z} / 3 Z)$. This proves Theorem 3 .

5. Proof of Theorem 4. The theorem will follow for an arbitrary number $n$ of boundary components if we can prove it for the closed case $(n=0)$ since for any $g$ and $n$ there is an epimorphism $M(F(g, n)) \rightarrow M(F(g, 0))([1, \S 4.1)]$. The idea for our proof is to use a certain matrix representation of $M=M(F(2,0))$ constructed by Jones [2]. By [1] $M$ is generated by Dehn Twists $T_{1}, \ldots, T_{5}$ and the Jones representation $J$ of $M$ satisfies

$$
\begin{aligned}
& J\left(T_{1}\right)=\left(\begin{array}{ccccc}
-1 & 0 & 0 & 0 & q \\
0 & -1 & 1 & 0 & 0 \\
0 & 0 & q & 0 & 0 \\
0 & 0 & 1 & -1 & 0 \\
0 & 0 & 0 & 0 & q
\end{array}\right), \\
& J\left(T_{2}\right)=\left(\begin{array}{ccccc}
q & 0 & 0 & 0 & 0 \\
0 & q & 0 & 0 & 0 \\
0 & q & -1 & 0 & 0 \\
1 & 0 & 0 & -1 & 0 \\
1 & 0 & 0 & 0 & -1
\end{array}\right), \\
& J\left(T_{3}\right)=\left(\begin{array}{ccccc}
-1 & 0 & 0 & q & 0 \\
0 & -1 & 1 & 0 & 0 \\
0 & 0 & q & 0 & 0 \\
0 & 0 & 0 & q & 0 \\
0 & 0 & 1 & 0 & -1
\end{array}\right)
\end{aligned}
$$

where $q$ is an indeterminate. Now note that if $(-1)^{k} q$ is a $k$ th root of 1 , then each of 
$(-1)^{k} J\left(T_{1}\right), \ldots,(-1)^{k} J\left(T_{5}\right)$ has order $k$ since an induction shows that for each $i \leq 5, T_{i}^{k}$ has the form

$$
(-1)^{k} I d+\left(q^{k-1}-q^{k-2}+\ldots+(-1)^{k-2} q+(-1)^{k-1}\right) W+\left(q^{k}-1\right) U
$$

for some matrices $W, U$. Thus we obtain a representation $J^{\prime}$ of $M / N$, where $N$ is the normal closure of $E^{k}$ by letting $(-1)^{k} q$ be a $k$ th root of 1 and putting $J^{\prime}\left(T_{i}\right)=(-1)^{k} J\left(T_{i}\right)$. Let $b$ be the bounding curve which is symmetric relative to $T_{1}, \ldots, T_{5}$. Then the Dehn twist about $b$ is $B=\left(T_{1} T_{2} T_{1}\right)^{4}$. Let $R=J^{\prime}\left(B T_{3} B T_{3}^{-1}\right)$. Then interchanging the 2nd and 4th rows and columns of $R$ gives a matrix $R^{\prime}$ having the form $\left(\begin{array}{ll}X & Y \\ O & t I\end{array}\right)$ where $X=X(q)$ is a $2 \times 2$ matrix, $t=q^{12}$ and $I$ is the $3 \times 3$ identity matrix. The characteristic polynomial of $X / q^{6}$ is

$$
x^{2}-x\left(q^{8}-2 q^{7}+q^{6}+2 q^{5}-2 q^{4}+2 q^{3}+q^{2}-2 q+1\right) / q^{4}+1 .
$$

One checks that for $k=4$, (with $q=i$ ) $X$ is a non-trivial parabolic; and that if $k=6$ (with $q=$ primitive cube root of 1 ) then $X$ has distinct eigenvalues which are not roots of unity (they have absolute values equal to $0 \cdot 10102 \ldots$ and $1 / 0 \cdot 10102 \ldots$ ). Thus in both cases we see that $R$ has infinite order. It follows that if $k$ is even and $3 \mid k$, then $R$ has infinite order. If $k=2(3 n \pm 1)>6$, then letting $q=\exp (4 \pi i n / k)$, we see that $R$ has infinite order by noticing that the absolute values of the eigenvalues of $X$ rapidly converge to $0 \cdot 10102 \ldots$ and $1 / 0 \cdot 10102 \ldots$ as $n \rightarrow \infty$. One similarly deals with the odd cases using $q=\exp (3 \pi i n / k)$ if $k=4 n \pm 1$ and $n$ is odd and $q=\exp ((3 n-1) \pi i / k)$ otherwise.

The author wishes to thank the referee for some useful comments.

\section{REFERENCES}

1. J. Birman, Braids, links and mapping class groups, (Princeton University Press, Annals of Math Studies \#82, 1975).

2. V. Jones, Hecke algebra representations of braid groups and link polynomials, Ann. Math. 126 (1987) 335-388. $225-242$.

3. D. Johnson, An abelian quotient of the mapping class group $I_{g}$, Math. Ann. 249 (1980)

4. D. Johnson, The structure of the Torelli Group II. Topology 24 (1985) 113-126.

5. D. Johnson, The structure of the Torelli Group I. Ann. Math. 118 (1983) 423-442.

6. W. B. R. Lickorish, A finite set of generators for the homeotopy group of a 2-manifold, Proc. Camb. Phil. Soc. 60 (1964) 769-778.

7. W. Magnus, A. Karrass and D. Solitar, Combinatorial group theory, (Dover, 1976).

8. J. Mennicke, Zur Theorie der Siegelschen Modulgruppe, Math. Ann. 157 (1965) 115-129.

9. M. Newman, Integral matrices, (Academic Press, 1972).

10. J. Powell, Two theorems on the mapping class group of surfaces, Proc. AMS 68 (1978) 347-350.

DEPARTMENT OF MATHEMATICS,

Brigham Young UnIVERSITY, Provo, Utah, 84602, U.S.A. 